sinage de Scuol) à une altitude de $2600 \mathrm{~m}$. Ces structures sont plus grandes que les microformes et se forment beaucoup plus lentement. Une autre sorte de polygones résulte d'un réseau de fissures. Ces fissures effectuent une congélation non proportionnée du sol et ensuite une migration d'un tas de pierres vers les fissures.

Les grandes formes des sols polygonaux se trouvent avant tout entre $2200 \mathrm{~m}$ et $2700 \mathrm{~m}$ d'altitude, les petites structures au-dessus de $2500 \mathrm{~m}$. Ces dernières sont moins fréquentes dans des positions plus basses parce qu'au printemps, les nuits de gel étant très fréquentes, la neige protège le sol de l'action des gelées nocturnes.

\title{
PROFIL HYPSOMÉTRIQUE DU FAITE DES ALPES CENTRALES
}

\section{MAX BOUËT}

La forme et la hauteur de la chaîne des Alpes jouent assurément un rôle important dans la distribution géographique, la direction et la fréquence du vent, et en particulier du fœhn. On sait en effet que ce dernier n'apparaît pas dans toutes les vallées en même temps ni avec la même intensité, ce dont le relief est certainement en grande partie responsable. De là l'idée d'étudier l'extension verticale de cet obstacle naturel et d'en évaluer l'importance. Contraint de fixer des limites, j'ai déterminé ici l'altitude moyenne des Alpes dans leur partie la plus élevée, située en majeure partie en Suisse ou le long de sa frontière méridionale.

\section{MÉTHODE}

Si l'on envisage les Alpes comme un obstacle aux vents, il est logique de rechercher l'altitude de leur plus haut profil submersible en le considérant sous l'angle de la statique des fluides. Me plaçant à ce point de vue et tenu de simplifier un problème topographique en lui-même compliqué, je me suis limité au faite alpin défini comme la ligne de partage des eaux entre les bassins du Rhône, du Rhin et du Danube d'une part, du Pô et de l'Adige d'autre part. J'ai donc laissé de côté les chaînes et massifs situés en-deça ou au-delà du dit faîte et qui par endroits peuvent pourtant lui être légèrement supérieurs, comme par exemple le groupe de la Jungfrau.

J'ai alors calculé, par sections, l'altitude moyenne du faite alpin entre le col du Petit-Saint-Bernard à la frontière franco-italienne et le passo di Rèsia (Reschenscheideck) à la frontière autro-italienne. La présence du puissant massif du Mont-Blanc où culmine la chaîne alpine justifiait l'extension du profil vers le Sud-Ouest, au-delà des Alpes italo-suisses proprement dites.

Le matériel cartographique utilisé fut le suivant: a) Au 1:50 000: Carte nationale de la Suisse; Carte du Mont-Blanc de Barbey/Imfeld/Kurz; Nouvelle carte de France; Carta delle zone turistiche d'Italia. b) Au 1:100 000: Carte topographique de la Suisse (Dufour); Carta del Istituto geografico militare.

C'est avant tout le premier de ces documents, la très remarquable «Carte nationale de la Suisse» au 1:50000 en cours de publication, qui a servi au pointage des nombreuses cotes d'altitude; les autres ont été consultés pour les parties de la crête situées entièrement en territoire français ou italien.

Après avoir relevé 921 cotes (1,6 par kilomètre) indiquées sur la carte ou déduites des isohypses, je les ai reportées graphiquement sous forme d'une ligne brisée reliant creux et sommets. Les distances horizontales nécessaires au calcul de l'altitude furent mesurées le long de 55 alignements sur lesquels vinrent se projeter les points cotés; ces alignements, longs de $8,6 \mathrm{~km}$. en moyenne, furent choisis de manière à s'adapter le mieux possible au tracé parfois très sinueux de la ligne de partage des eaux. Cette mesure des distances par projection est simple et précise; elle substitue à la coupe verticale réelle un profil contracté dans le sens horizontal sans toutefois modifier l'alti- 
tude moyenne. D'ailleurs la longueur effective de la ligne de faîte fut mesurée au curvimètre en suivant ses moindres sinuosités, mais par larges tranches correspondant aux sections du tableau 1.

La surface du profil vertical ainsi dressé fut alors planimétrée pour permettre le calcul de l'altitude moyenne. L'ensemble fut subdivisé en 29 sections choisies entre les principaux cols de manière à comprendre un crête d'altitude aussi uniforme que possible. Ces sections sont en général plus courtes dans la partie occidentale très accidentée, plus longues à l'Est du Gothard où la crête présente de moins grandes variations d'altitude.

L'erreur relative de planimétrie étant de l'ordre de 0,001 , elle conduit à une erreur d'altitude inférieure au demi-décamètre. Toutefois le tracé en ligne brisée reliant les points cotés diffère naturellement du profil réel de la crête, malgré le grand nombre de cotes relevées sur les cartes. Après un essai particulier qui me permit de comparer la photographie d'une crête et sa représentation polygonale, j'ai admis qu'il y avait compensation plus ou moins parfaite entre les écarts positifs et négatifs de surface du profil géométrique par rapport à la silhouette naturelle impossible à suivre dans le détail. Au reste, à l'échelle du 1:50 000, le tracé de la ligne de faîte est par endroits légèrement incertain et de plus la pente des parties rocheuses est pratiquement inconnue. L'approximation de la ligne brisée allant des creux aux saillies repérables sur la carte est donc la seule possible.

J'admets en définitive que l'altitude moyenne est connue avec une erreur inférieure au décamètre. Les valeurs du tableau 1 sont arrondies au décamètre entier le plus proche.

\section{LA LIGNE DE FAITE}

C'est, je le rappelle, la ligne de partage des eaux des bassins fluviaux du Nord et du Sud des Alpes. Son développement total mesure $569 \mathrm{~km}$. entre le col du PetitSaint-Bernard et celui de Rèsia, ces deux points étant éloignés en ligne droite de 307,8 $\mathrm{km}$. seulement. Voici, esquissé à grands traits, le jalonnement de la crête dont les 29 sections figurent au tableau 1 avec leurs données altimétriques.

Groupe du Mont-Blanc. Du col du Petit-Saint-Bernard à celui de la Seigne, le faîte suit la frontière franco-italienne en passant par le Bec des Rousses, le Mont de Mirande et la Pointe Léchaud. Il aborde alors le massif du Mont-Blanc proprement dit en suivant également la frontière politique jalonnée par les Aiguilles des Glaciers, de Tré-la-Tête et de Bionassay, le Mont-Blanc, le Mont-Maudit, l'arête des Jorasses et le Dolent. Bien que le faîte culmine ici au sommet du Mont-Blanc (4807 m.), cette partie ne présente pas l'altitude moyenne la plus grande par suite de la structure très découpée du massif.

Alpes pennines. Dès le Dolent la ligne de crête coïncide avec la frontière italosuisse, sauf un écart de $300 \mathrm{~m}$ au lac du Saint-Bernard. Après l'ensellement des cols Ferret et de celui du Grand-Saint-Bernard, elle se relève lentement en passant par le Vélan, le Mont-Gelé, la Singla, la Dent d'Hérens, le Cervin, le Breithorn, le MontRose, le Joderhorn et le Portjengrat. Le massif du Mont-Rose, en raison de sa forte massiveté, présente l'altitude moyenne la plus élevée $(4140 \mathrm{~m}$.) de toute la chaîne. A partir du point coté $3653,8 \mathrm{~m}$. (Carte nat. de la Suisse), la ligne de partage des eaux quitte la frontière politique, escalade le Weissmies et le Lagginhorn de plus de 4000 m., gagne le Fletschhorn et le Spitzhörnli pour descendre ensuite au col du Simplon à $2005 \mathrm{~m}$.

Alpes lépontines. On retrouve la frontière italo-suisse à partir d'un épaulement (3403 m.) du Monte-Leone, puis on passe par le Helsenhorn, l'Ofenhorn, le Blinnenhorn et le Grieshorn.

Groupe $d u$ Gothard. La crête quitte la frontière politique, franchit le Nufenenpass, le P. Gallina, le P. Rotondo et le P. Lucendro pour s'abaisser au col du Gothard 
(2108 m.) ; elle remonte au P. Centrale, sépare le val Cadlimo du val Piora et aboutit au col du Lukmanier (passo di Lucomagno).

Groupe de l'Adula. A partir du Lukmanier, la ligne de partage des eaux présente un tracé extrêmement sinueux et compliqué; elle passe par le Scopi, le P. Medel, le P. Terri, traverse le massif de l'Adula, se creuse au col du San-Bernardino puis à celui du Splügen; elle emprunte le territoire italien à l'Ouest de la valle di Lei, du P. Timun à la Cima di Lago, limite au Nord le bassin de la Maira, franchit le Septimer, puis descend au col de la Maloja au point le plus bas des Alpes suisses proprement dites (1815 m.).

Alpes rhétiques. La ligne de faîte s'élève une dernière fois au-dessus de $4000 \mathrm{~m}$. dans le massif de la Bernina, descend au col du même nom (plus exactement au Lago Bianco à $2234 \mathrm{~m}$.), passe de nouveau en Italie sur la rive droite de Spöl du P. Valnera jusqu'au passo di Fraéle; de là elle est jalonnée par: M. Forcola, P. Dössradond, pass del Fuorn, P. Starlex, P. Sesvenna. Elle atteint enfin le passo di Rèsia (Reschenscheideck) aux sources de l'Adige, à la frontière austro-italienne; le col lui-même se trouve sur territoire italien, à environ $3 \mathrm{~km}$. de la frontière suisse. La section comprise entre le col de Foscagno et celui de Fraéle est la plus basse de toutes avec $2560 \mathrm{~m}$. d'altitude moyenne.

\section{ALTITUDES MOYENNES}

Le tableau 1 résume tout ce que le procédé de calcul ci-dessus exposé permet d'obtenir et n'exige pas grand commentaire. Il contient d'abord la liste des sections allant de col en col avec l'altitude de ceux-ci; viennent ensuite les longueurs effectives des sections mesurées au curvimètre, puis leur altitude moyenne arrondie au décamètre. Enfin figure pour chaque section le point le plus bas qui est, à une exception près, l'un des deux cols terminaux, ainsi que le point le plus haut.

Il est intéressant de remarquer que la partie occidentale des Alpes, à l'Ouest du Gothard, est d'environ $400 \mathrm{~m}$. plus élevée que la partie orientale. On remarquera aussi que les grandes entailles de la chaîne vont se creusant d'Ouest en Est, comme le montre la liste des cols suivants: Grand-Saint-Bernard 2469 m., Simplon 2005 m., Lucomagno 1916 m., Maloja 1815 m. et Rèsia 1510 m.

Enfin le calcul donne pour l'ensemble de la chaîne, du Petit-Saint-Bernard au passo di Rèsia, l'altitude moyenne de $3040 \mathrm{~m}$.; ainsi, entre les limites précitées et en s'en tenant au faîte défini par l'hydrographie, le rempart alpin s'élève en moyenne à $3000 \mathrm{~m}$. d'altitude. Doublée au Nord par la longue et haute chaîne des Alpes bernoises et par celle des Alpes glaronnaises et grisonnes, cette imposante barrière sépare deux régions distinctes du monde végétal tout comme deux zones climatiques ayant chacune leurs traits caractéristiques; elle introduit dans l'allure du temps météorologique de l'Europe centrale des modifications profondes et une dissymétrie presque constante entre les deux versants.

Cette dissymétrie se traduit dans le régime pluviométrique comme l'ont très bien montré MM. BIDER et Thams ${ }^{1}$ ainsi que l'auteur ${ }^{2}$. En outre la haute barrière alpine introduit d'importantes modifications dans le champ de pression puisqu'elle sépare deux bassins aériens d'environ $3000 \mathrm{~m}$. de profondeur (Plateau suisse et plaine du Pô) et de température en général différente; il s'ensuit un renforcement fréquent du gradient transalpin, soit par courant du Nord-Ouest, soit par situation de fœhn. Enfin l'altitude élevée du faîte alpin de long de la vallée du Rhône explique probablement dans une certaine mesure la rareté relative du fœhn en Valais, fait que j'ai relevé ailleurs déjà 3 et au sujet duquel de nouvelles recherches sont en cours.

Il serait intéressant que le calcul hypsométrique appliqué ici à la partie centrale et la plus élevée de la chaîne fût étendu aux Alpes françaises d'une part et autrichiennes de l'autre, afin que l'on puisse embrasser dans un seul profil l'ensemble de l'arc alpin. Si du côté français le faîte est défini sans ambiguïté, à l'Est de la Suisse 
il ne l'est plus au-delà des Alpes du Zillertal où la chaîne des Tauern, quoique située à l'intérieur du bassin danubien, constitue en fait l'obstacle principal, plus important que les Alpes carniques plus basses qui ferment ce bassin au Sud.

Tableau I. Faîte des Alpes. Altitudes

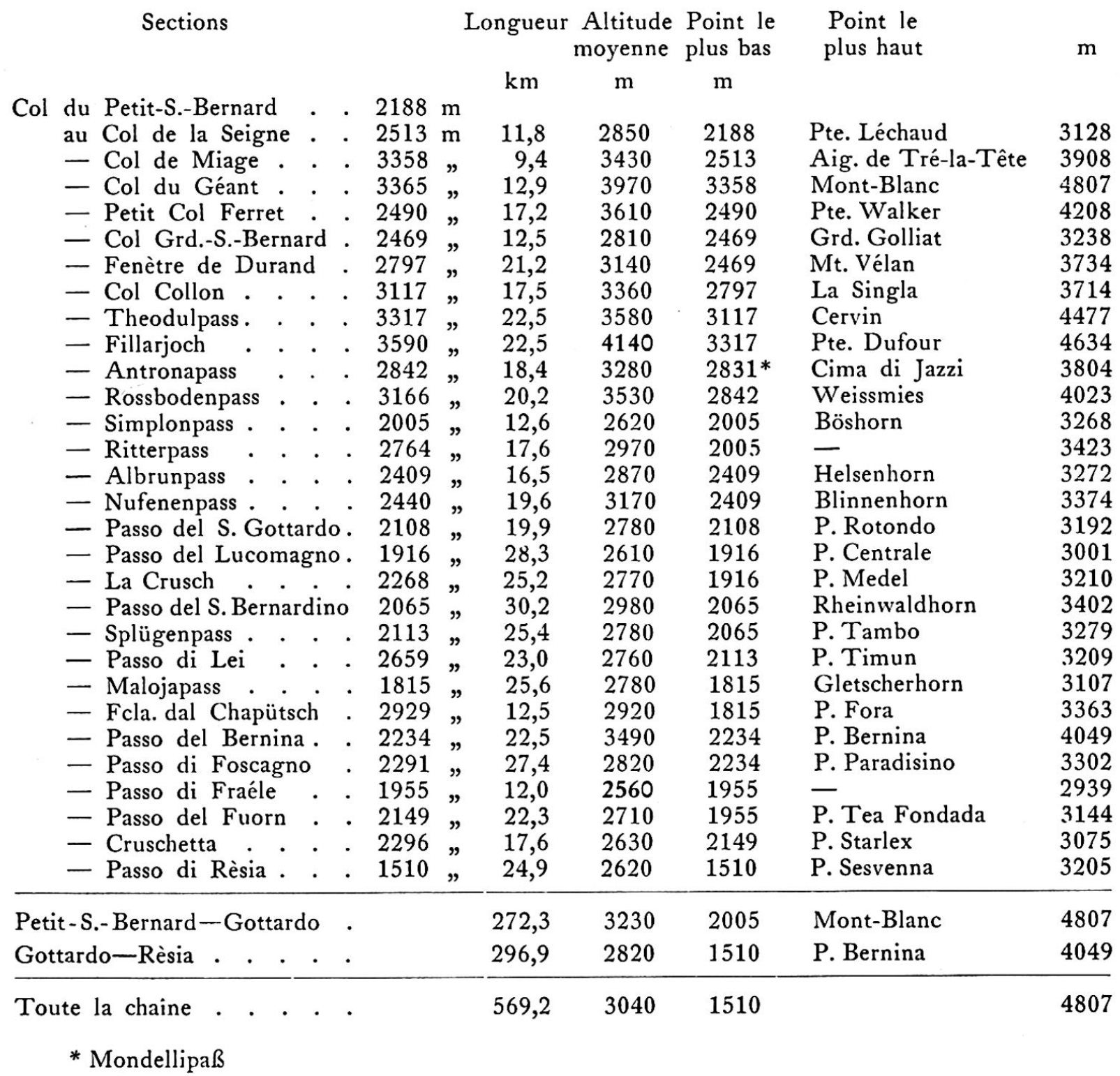

ARTICLES CITÉS: ${ }^{1}$ BIDER, M. und ThAMs, J. C.: Das Niederschlagsregime nord- und südwärts der Alpen auf Grund zehnjähriger Pluviographenregistrierungen. Archiv f. Met., Geoph. u. Bioklimat., Bd. 3, 1951. $-{ }^{2}$ BouĒT, M.: La dissymétrie des régimes pluviaux au Nord et au Sud des Alpes suisses. Geofisica pura e applicata, vol. 19, 1951. - ${ }^{3}$ BouËr, M.: Le fœhn en Valais. Bull. Murithienne, fasc. 68, 1951.

\section{HYPSOMETRISCHES PROFIL ÜBER DIE WASSERSCHEIDE DER ZENTRALALPEN}

Durch Planimetrie des Vertikalschnittes wird die mittlere Höhe der Wasserscheide zwischen Rhone-, Rhein- und Donaugebiet einerseits, Po- und Etschgebiet andererseits streckenweise berechnet, und zwar vom Kleinen St. Bernhard bis zum Reschenscheideck. Die mittlere Höhe der Zentralalpen beträgt $3000 \mathrm{~m}$. 


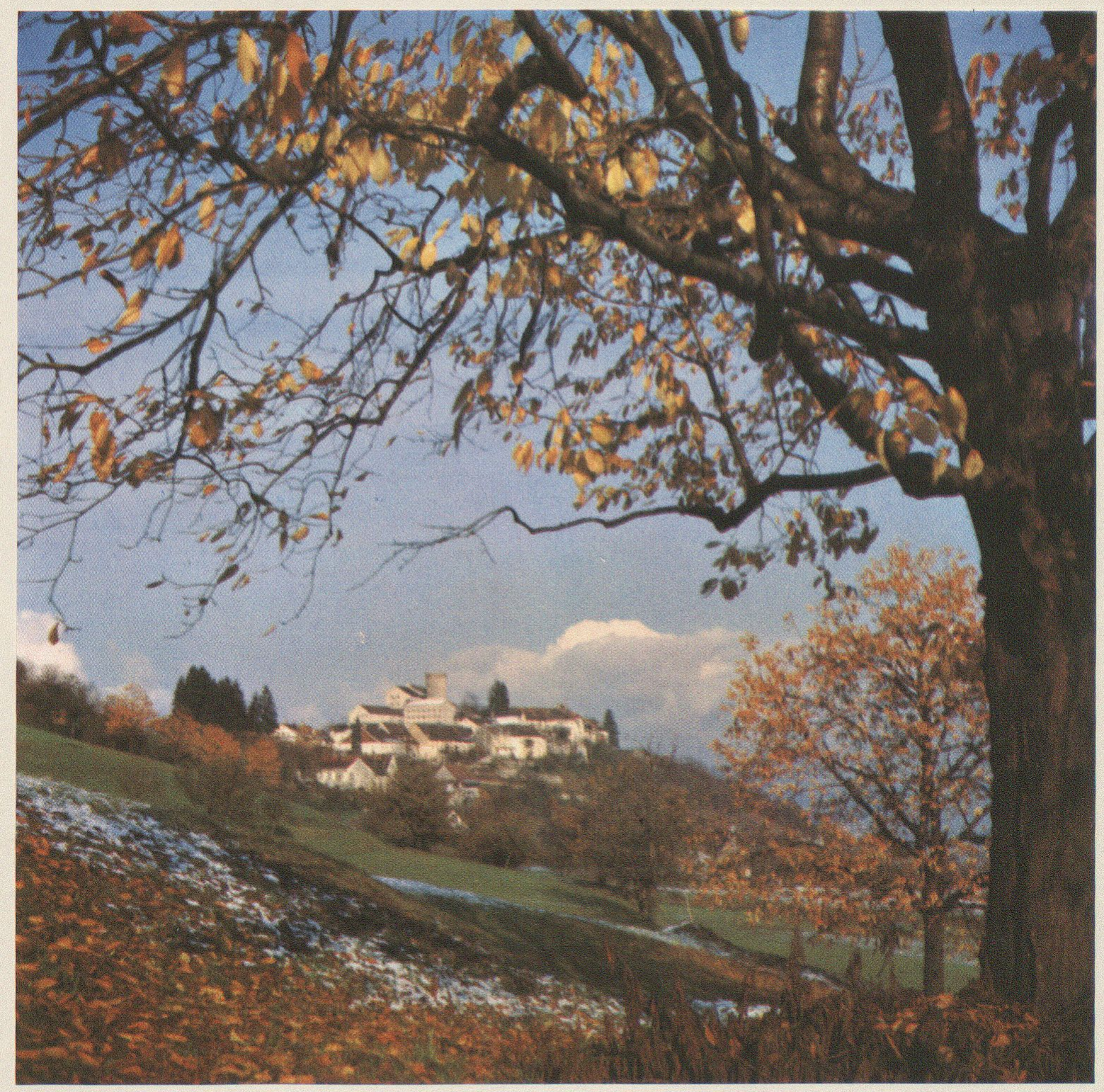

Photo K. Beyeler, Goldbach

Regensberg. Verträumt schaut das alte Städtchen vom östlichen Sporn des Lägernrückens in die Landschaft hinaus. - Blick auf Oberburg mit Wehrturm. 